01

\title{
Образование пузырька на гидрофобной поверхности
}

\author{
() С.И. Кошоридзе, Ю.К. Левин \\ Институт прикладной механики РАН, \\ 125040 Москва, Россия \\ e-mail: koshoridze-semen@yandex.ru
}

Поступило в Редакцию 30 апреля 2019 г.

В окончательной редакции 18 декабря 2019 г.

Принято к публикации 15 января 2020 г.

\begin{abstract}
Исследовано образование заряженного равновесного нанопузырька на гладкой гидрофобной поверхности раздела вода-твердое тело. Даны выражения для изменения энергии Гиббса системы с учетом капиллярной формулы Кельвина, электростатической энергии двойного электрического слоя на границе пузырь-вода и поверхностной энергии. Показано, что график зависимости энергии Гиббса от радиуса нанопузырька имеет минимум, что указывает на возможность самопроизвольной генерации данных наноструктур. Исследована зависимость энергии Гиббса от контактного угла, а также солености и коэффициента поверхностного натяжения воды. Рассчитан поверхностный заряд, необходимый для стабильности нанопузырей.
\end{abstract}

Ключевые слова: поверхностный нанопузырь, двойной электрический слой, энергия Гиббса, капиллярная формула Кельвина, поверхностное натяжение воды.

DOI: 10.21883/JTF.2020.06.49272.182-19

В мире растет число экспериментальных исследований поверхностных нанопузырьков в связи с их широким применением в различных областях науки и техники [1], однако до сих пор не ясны ни термодинамика, ни аномально большие времена жизни данных структур.

В $[2,3]$ была предпринята попытка термодинамического расчета образования равновесных нанопузырьков на границе раздела вода-гидрофобная поверхность (рис. 1) без учета заряда. В [4] справедливость данной теории была подвержена критике. В настоящей работе проводится термодинамический анализ образования равновесного пузырька на гидрофобной поверхности и исправлены неточности, допущенные в [2,3].

Будем считать, что образование пузырька в системе воздух-вода-гидрофобная поверхность происходит в изобарно-изотермических условиях. Изменение термодинамического потенциала Гиббса $\Delta G$ при образовании заряженного пузырька на гладкой границе раздела твердое тело-жидкость (вода) записывается как сумма поверхностной $\Delta G_{s}$ и объемной $\Delta G_{v}$ составляющих [2,3]. В нашей теории учтем, что пузырь имеет заряд [5], поэтому добавляется составляющая $\Delta G_{e}$, связанная с энергией двойного электрического слоя:

$$
\Delta G=\Delta G_{s}+\Delta G_{v}+\Delta G_{e} .
$$

Образование пузырька будет термодинамически возможно только при выполнении условия $\Delta G<0$.

Изменение энергии Гиббса при формировании двойного электрического слоя на границе пузырь-вода отрицательно: $\Delta G_{e}<0$. Согласно [5], отрицательные ионы гидроксида переходят из воды на пузырь, уменьшая потенциал Гиббса системы. Пузырь приобретает отрицательный заряд, а в воде вокруг него образуется положительная область объемного заряда, ширина которого зависит от концентрации солей в воде. Изменение свободной энергии Гиббса $\Delta G_{e}$ при образовании двойного слоя равно интегралу [6]

$$
\Delta G_{e}=-A_{l g} \int_{0}^{\psi_{0}} \sigma(\psi) d \psi,
$$

где $\sigma(\psi)$ - модуль плотности поверхностного заряда пузырька, $\psi$ - модуль потенциала поверхности раздела пузырька, площадь границы раздела вода-пузырек

$$
A_{l g}=2 \pi R_{b}^{2}(1-\cos \omega)=2 \pi R_{b}^{2}(1+\cos \theta),
$$

$\psi_{0}$ - электрокинетический потенциал пузырька, $\omega-$ полярный угол, $\theta=180^{\circ}-\omega-$ так называемый характерный контактный угол между гладкой поверхностью и пузырьком (рис. 1).

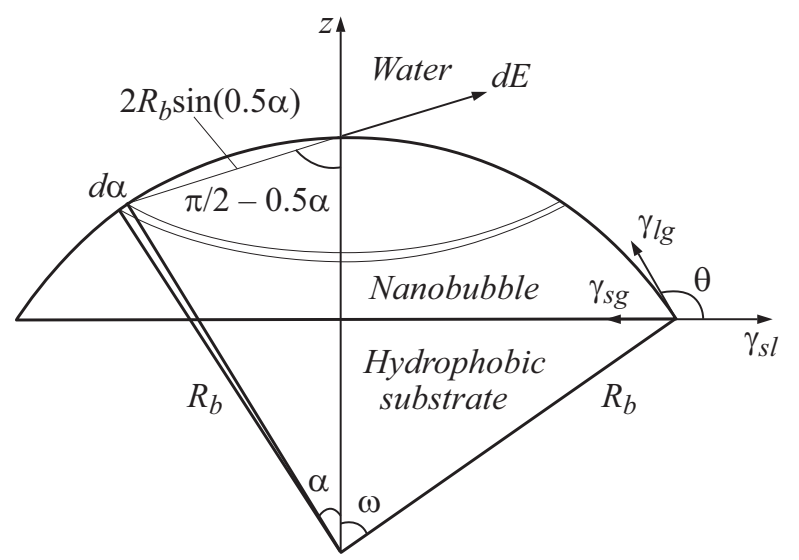

Рис. 1. Нанопузырь на гладкой поверхности раздела вода-твердое тело. 
Выражение (2) можно представить как энергию сферического конденсатора:

$$
\Delta G_{e}=-\frac{A_{l g}^{2} \sigma^{2}}{2 C}
$$

где $\sigma \equiv \sigma\left(\psi_{0}\right)$. Для простоты расчетов будем считать, что потенциал $\psi$ вокруг пузырька обратно пропорционален радиусу $R_{b}$ пузырька. Тогда применима формула для емкости сферического конденсатора радиуса $R_{b}$ (рис. 1 ):

$$
C=\frac{A_{l g}}{R_{b}^{2}} \frac{\varepsilon_{0} \varepsilon R_{b}\left(R_{b}+L_{D}\right)}{L_{D}}
$$

- электрическая емкость пузыря, представляемого как конденсатор с обкладками в виде шаровых сегментов и расстоянием между обкладками, равным длине Дебая:

$$
L_{D}=\sqrt{\frac{\varepsilon \varepsilon_{0} k_{\mathrm{B}} T}{2 e^{2} N_{\mathrm{A}} c}},
$$

где $c$ - мольная концентрация солей в воде (для простоты рассматриваем соль $\mathrm{NaCl}) ; e, k_{\mathrm{B}}$ и $N_{\mathrm{A}}-$ элементарный заряд, постоянная Больцмана и число Авогадро соответственно; $T$ - абсолютная температура; $\varepsilon$ - диэлектрическая проницаемость воды, $\varepsilon_{0}-$ электрическая постоянная.

Поверхностная составляющая энергии Гиббса $\Delta G_{s}$ вычисляется по формуле [2]

$$
\Delta G_{s}=A_{l g} \gamma_{l g}+A_{s g}\left(\gamma_{s g}-\gamma_{s l}\right)
$$

где $\gamma_{l g}, \gamma_{s g}$ и $\gamma_{s l}$ - коэффициенты поверхностного натяжения на границе жидкость-газ, твердое тело-газ и твердое тело-жидкость соответственно

$$
A_{s g}=\pi R_{b}^{2} \sin ^{2} \omega=\pi R_{b}^{2} \sin ^{2} \theta
$$

- площадь границы раздела твердое тело-пузырек. Контактный угол $\theta$ между твердым телом и водой удовлетворяет формуле Юнга:

$$
\cos \theta=\frac{\gamma_{s g}-\gamma_{s l}}{\gamma_{l g}}
$$

Формулы (3), (7)-(9) дают

$$
\Delta G_{s}=\pi R_{b}^{2} \gamma_{l g}\left(2+2 \cos \theta+\sin ^{2} \theta \cos \theta\right) .
$$

Газы в пузырьке находятся в равновесии с тем же газом, растворенными в воде, т. е. химический потенциал каждой компоненты (вода, азот, кислород) в газовой фазе и в растворенном состоянии равны. Это означает, что массообмена между водой и пузырьком не происходит. Будем считать, что объемная часть гиббсовского потенциала жидкой фазы при образовании пузырька не меняется [2,3]. Объемную часть изменения потенциала Гиббса газовой фазы (и всей системы) можно записать как разность суммарных химических потенциалов компонентов над плоской (атмосфера) и вогнутой (пузырь) поверхностями раздела фаз вода-воздух, используя соотношение Кельвина (считается, что вода контактирует с атмосферой, а пузырь находится на малой глубине):

$$
\begin{aligned}
\Delta G_{v} & =\sum_{i=1}^{3} \Delta G_{v i}=R T \sum_{i=1}^{3} n_{i} \ln \left(\frac{p_{g i}}{p_{a i}}\right) \\
& =-\frac{2 \gamma_{l g}}{\rho R_{b}} \sum_{i=1}^{3} M_{i} n_{i}<0 .
\end{aligned}
$$

Здесь $n_{i}$ - число молей $i$-ой компоненты в пузырьке, $R$ - универсальная газовая постоянная, $\rho$ - плотность воды, $M_{i}$ - молярная масса той же компоненты $(i=1,2,3$ - для воды, азота, кислорода соответственно); $p_{g i}$ - парциальное давление $i$-ой компоненты в пузырьке, связанное с парциальным давлением той же компоненты в атмосфере $p_{a i}$ соотношением Кельвина:

$$
p_{g i}=p_{a i} \exp \left(-\frac{2 \gamma_{l g} M_{i}}{\rho R_{b} R T}\right) .
$$

Следует отметить, что в [2] давление внутри пузыря приравнивалось к давлению паров воды без учета давления азота и кислорода, а вместо молярного объема воды $\frac{M_{1}}{\rho}$ фигурировал объем пузырька.

Число молей $n_{i}$ определим из уравнения состояния идеального газа

$$
n_{i}=\frac{V_{b} p_{g i}}{R T} .
$$

Здесь

$$
V_{b}=\frac{\pi}{3} R_{b}^{3}(\cos \omega+2)(1-\cos \omega)^{2}
$$

- объем поверхностного нанопузырька.

С учетом (11)-(14) для объемной части изменения гиббсовского потенциала получим

$$
\begin{aligned}
\Delta G_{v} & =-\frac{2 \gamma_{l g} \pi R_{b}^{2}}{3 R T \rho}(1-\cos \omega)^{2}(2+\cos \omega) \sum_{i=1}^{3} p_{a i} M_{i} \\
& \times \exp \left(-\frac{2 \gamma_{l g} M_{i}}{\rho R_{b} R T}\right) .
\end{aligned}
$$

Как отмечено выше, равновесные пузыри имеют поверхностный заряд, поэтому в соотношении Юнга-Лапласа в отличие, например, от других работ по термодинамике поверхностных нанопузырьков $[7,8]$, в настоящей работе учтено электростатическое давление $p_{e}$, компенсирующее избыточное капиллярное давление [9]:

$$
\sum_{i=1}^{3} p_{g i}+p_{e}=p_{a}+\frac{2 \gamma_{l g}}{R_{b}} .
$$

Осталось найти связь между радиусом $R_{b}$ и плотностью $\sigma$ поверхностного заряда и рассчитать, какой заряд пузырька необходим для установления механического равновесия (16). Для упрощения физической картины 


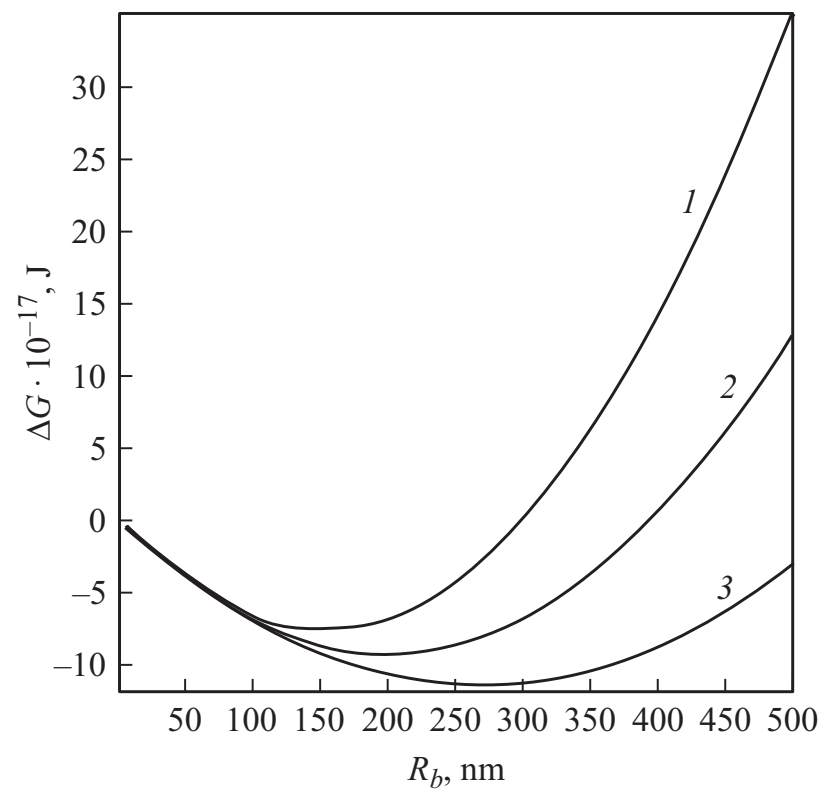

Рис. 2. Изменение потенциала Гиббса при образовании поверхностного нанопузырька $\Delta G$ как функция радиуса $R_{b}$ при различных значениях контактного угла $\theta$. Расчетные параметры: $c=0.01 \frac{\mathrm{mol}}{\mathrm{l}}, M_{1}=0.018 \frac{\mathrm{kg}}{\mathrm{mol}}, M_{2}=0.028 \frac{\mathrm{kg}}{\mathrm{mol}}$, $M_{3}=0.032 \frac{\mathrm{kg}}{\mathrm{mol}}, \quad p_{a}=1 \mathrm{~atm}, \quad T=293 \mathrm{~K}, \quad p_{a 1}=0.34 \cdot 10^{3} \mathrm{~Pa}$, $p_{a 2}=0.8 \cdot 10^{5} \mathrm{~Pa}, \quad p_{a 3}=0.2 \cdot 10^{5} \mathrm{~Pa}, \quad \gamma_{l g}=0.072 \frac{\mathrm{J}}{\mathrm{m}^{2}}, \quad \varepsilon=81$; $1-\theta=158^{\circ}, 2-\theta=160^{\circ}, 3-\theta=162^{\circ}$.

допустим, что заряд равномерно распределен на поверхности пузырька, а электростатическое давление $p_{e}$ однородно по всей его поверхности. Найдем электростатическое давление в пузырьке $p_{e}$. Для этого разобьем шаровой слой на полосы площадью

$$
d S=2 \pi R_{b}^{2} \sin \alpha d \alpha .
$$

Каждая полоса создает в вершине пузыря электростатическое поле с вертикальной составляющей, равной

$$
d E_{z}=\frac{\cos (0.5 \alpha) \sigma d \alpha}{4 \varepsilon \varepsilon_{0}} .
$$

После интегрирования по углу $\alpha$ от 0 до $\omega$ для электростатического давления в вершине пузырька получим

$$
p_{e}=\sigma E_{z}=\frac{\sigma^{2} \sin (0.5 \omega)}{2 \varepsilon \varepsilon_{0}}=\frac{\sigma^{2} \cos (0.5 \theta)}{2 \varepsilon \varepsilon_{0}} .
$$

Если подставить (19) в (16), можно посчитать $\sigma$ и электростатическую энергию $\Delta G_{e}(2)$.

Таким образом, получены все слагаемые для изменения энергии Гиббса $\Delta G(1)$ при образовании заряженного поверхностного нанопузырька. Надо еще раз подчеркнуть, что нанопузырь равновесный: он соответствует условию динамического равновесия с окружающей средой (16), а давление газа внутри него соответствует равновесному согласно (12).
На рис. 2 представлена зависимость энергии Гиббса образования равновесного нанопузырька $\Delta G$ от его радиуса $R_{b}$. Минимуму на данной кривой соответствуют радиусы пузырьков $R_{b \text { min }}$ с максимальной вероятностью образования.

Из графиков рис. 2 следует, что с увеличением контактного угла $\theta$ значение $R_{b \text { min }}$ и глубина минимума функции $\Delta G\left(R_{b}\right)$ возрастают.

На рис. 3 представлена зависимость $\Delta G\left(R_{b}\right)$ при различных значениях солености воды. С увеличением концентрации солей $c$ уменьшается длина Дебая $L_{D}$, растет емкость двойного слоя $C$ и при заданном поверхностном заряде $\sigma$, согласно (4), уменьшается доля отрицательной электростатической составляющей энергии Гиббса $\Delta G_{e}$ (см. (1)). В результате графики $\Delta G\left(R_{b}\right)$ смещаются вверх, и условия образования нанопузырьков ухудшаются. На рис. 4 представлена зависимость $\Delta G\left(R_{b}\right)$ при различных значениях коэффициента поверхностного натяжения воды $\gamma_{l g}$. Если величина $\gamma_{l g}$ уменьшается изза наличия в воде поверхностно-активных веществ, то это вызывает уменьшение сжимающего лапласовского давления $p_{e}$ и соответственно уменьшение плотности поверхностного заряда $\sigma$ (см. (6)) и модуля электростатической составляющей энергии Гиббса $\Delta G_{e}$. Как следствие - глубина минимума функции $\Delta G\left(R_{b}\right)$ уменьшается.

На всех приведенных на рис. 2-4 графиках при изменении радиуса $R_{b}$ пузырька контактный угол $\theta$ остается постоянным. Этим наша теория отличается от так называемой теории пининга $[1,10]$. Пининг — это за-

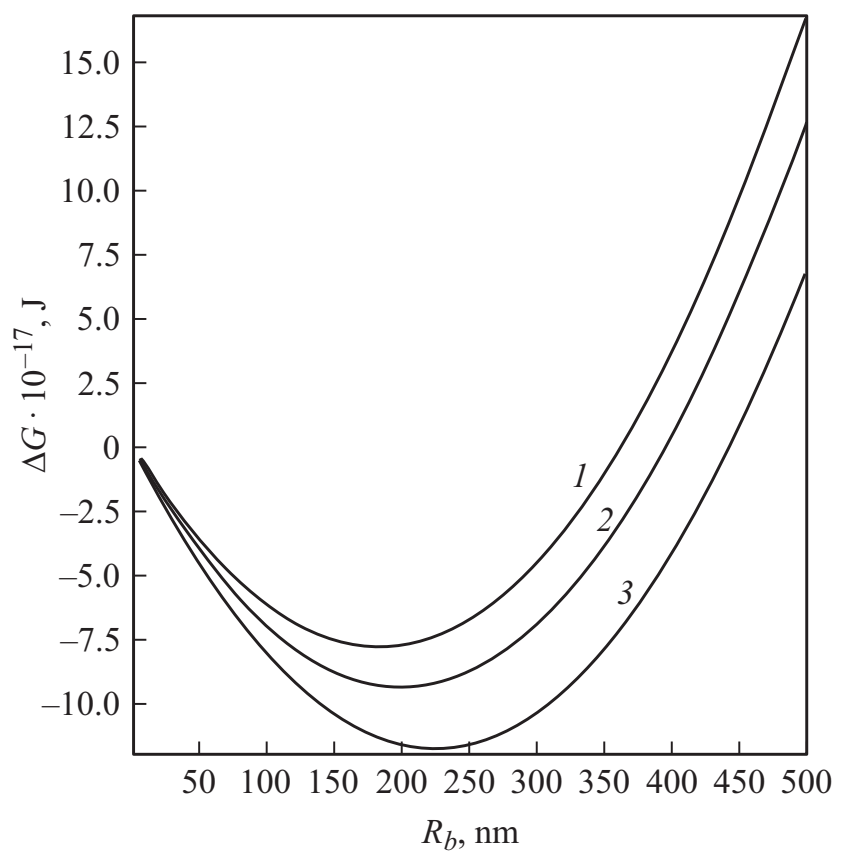

Рис. 3. Изменение потенциала Гиббса при образовании поверхностного нанопузырька $\Delta G$ как функция радиуса $R_{b}$ при разных значениях солености воды $c$. Расчетные параметры: $\theta=160^{\circ}$, остальные такие же, что на рис. $2.1-c=0.012 \frac{\mathrm{mol}}{1}$, $2-c=0.010 \frac{\mathrm{mol}}{1}, 3-c=0.008 \frac{\mathrm{mol}}{\mathrm{l}}$. 


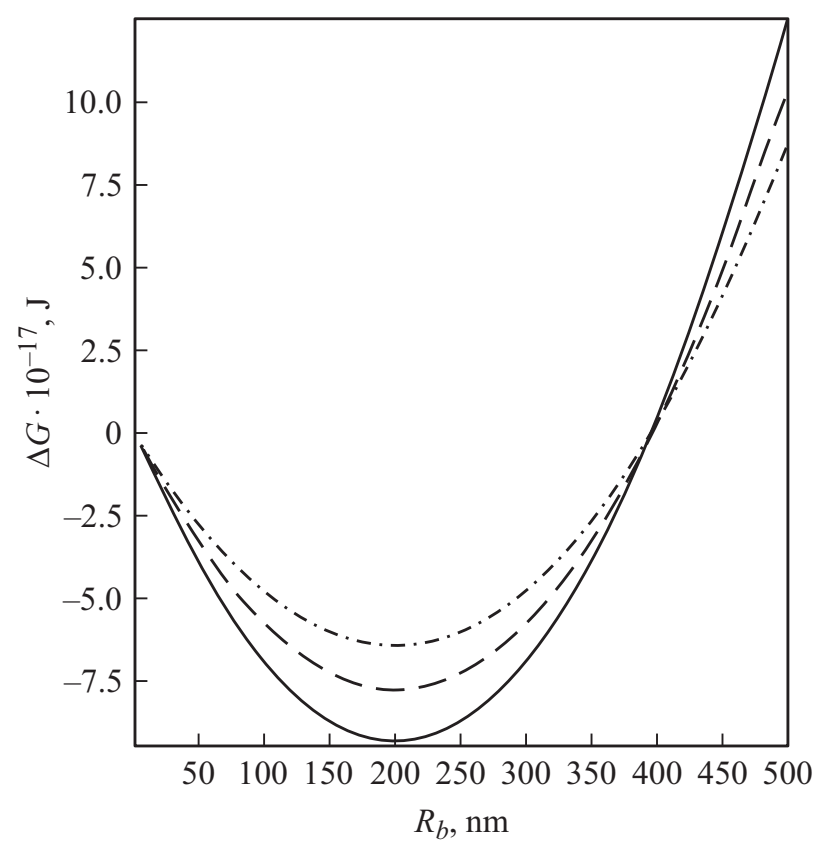

Рис. 4. Изменение потенциала Гиббса при образовании поверхностного нанопузырька $\Delta G$ как функция радиуса $R_{b}$ при разных значениях коэффициента поверхностного натяжения воды $\gamma_{l g}$. Расчетные параметры: $\theta=160^{\circ}$, остальные такие же, что на рис. 2. Сплошная линия $-\gamma_{l g}=0.072 \frac{\mathrm{J}}{\mathrm{m}^{2}}$, штриховая линия $-\gamma_{l g}=0.060 \frac{\mathrm{J}}{\mathrm{m}^{2}}$, штрихпунктирная линия $\gamma_{l g}=0.050 \frac{\mathrm{J}}{\mathrm{m}^{2}}$.

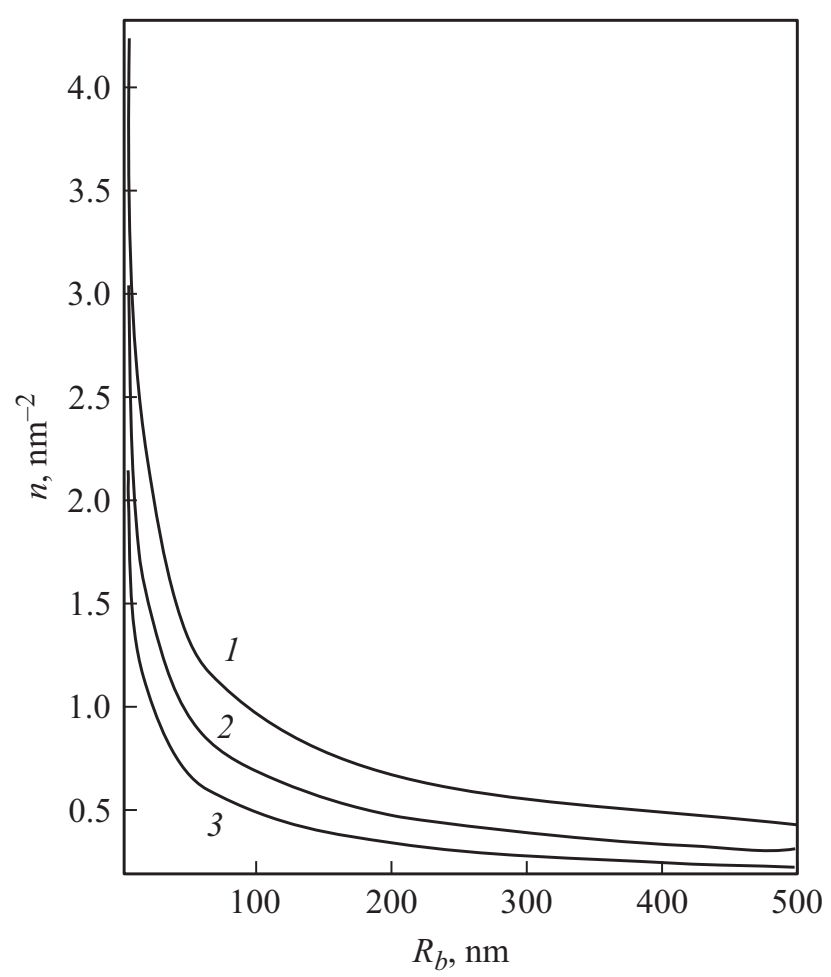

Рис. 5. Зависимость количества элементарных зарядов, приходящихся на единицу площади поверхностного нанопузырька $n$ от его радиуса $R_{b}$, для различных значений контактного угла $\theta$. Расчетные параметры такие же, как на рис. 2. $1-\theta=170^{\circ}$, $2-\theta=160^{\circ}, 3-\theta=140^{\circ}$. крепление границы между подложкой и поверхностным нанопузырьком: при росте пузырька контактный угол уменьшается, лапласовское давление растет и пузырь стабилизируется. Пининг нанопузырька обеспечивается за счет шероховатости подложки. В изложенной выше теории устойчивость пузырька достигается его поверхностным зарядом.

На рис. 5 представлена зависимость числа элементарных зарядов $e$ на единицу площади нанопузырька $n=\sigma / e$ от радиуса $R_{b}$ при различных значениях контактного угла $\theta$. С ростом контактного угла, согласно (19), электростатическое давление $p_{e}$ падает, и для сохранения давления нужно увеличивать плотность поверхностного заряда $\sigma$. При типичной концентрации ПН в воде [5] $10^{14} \mathrm{~m}^{-3}$, имеющих диаметр $R_{b}=100 \mathrm{~nm}$, получим, что для стабилизации ПН требуется примерно $10^{18}$ ионов гидроксида на $1 \mathrm{~m}^{3}$ воды. Если учесть, что нейтральному показателю кислотности $p H=7$ при комнатной температуре соответствует концентрация ионов гидроксида $6 \cdot 10^{19} \mathrm{~m}^{-3}$, можно сделать вывод, что для обеспечения стабильности поверхностных нанопузырьков адсорбированными ионами $\mathrm{OH}^{-}$, вода должна быть нейтральной или щелочной.

Следует отметить, что дополнительными факторами, обеспечивающими стабильность поверхностных нанопузырьков, могут являться:

1. Отрицательное значение линйного натяжения на границе раздела твердой, газообразной и жидкой фаз [11];

2. Отрицательное расклинивающее давление, возникающее внутри пузырька вблизи границы трех фаз, вызванное ван-дер-ваальсовым взаимодействием молекул воды и подложки, увеличивающее контактный угол $\theta$ [12].

В последующих работах авторов предполагается провести учет влияния зарождения и стабильности нанопузырьков.

\section{Основные результаты}

1. На гладкой гидрофобной поверхности возможно спонтанное образование поверхностных нанопузырьков, стабилизированных электрическим зарядом. Показано, что зависимость изменения энергии Гиббса нанопузырька от его радиуса $\Delta G\left(R_{b}\right)$ имеет минимум.

2. С ростом характерного контактного угла между нанопузырьком и гидрофобной подложкой $\theta$ и уменьшением солености воды $c$ увеличивается вероятность образования нанопузырька и диапазон допустимых значений его радиуса $R_{b}$.

3. Уменьшение коэффициента поверхностного натяжения воды $\gamma_{l g}$ понижает вероятность образования заряженных равновесных нанопузырей.

4. С увеличением контактного угла $\theta$ нанопузырька возрастает необходимая для его стабилизации плотность заряда $\sigma$. Показано, что водная среда должна быть нейтральной или щелочной. 


\section{Конфликт интересов}

Авторы заявляют, что у них нет конфликта интересов.

\section{Список литературы}

[1] Sun Y., Xie G., Peng Y., Xia W., Sha J. // Colloid. Surface. A. 2016. Vol. 495. P. $176-186$

DOI: $10.1016 /$ j.colsurfa.2016.01.050

[2] Colaco R., Serro A.P., Saramago B. // Surf. Sci. 2009. Vol. 603. P.2870-2873. DOI: 10.1016/j.susc.2009.07.034

[3] Colaco R., Serro A.P., Saramago B. // Surf. Sci. 2010. Vol. 604. P. 478-479. DOI: 10.1016/j.susc.2009.12.002

[4] Seddon J.R.T., Zandvliet H.J.W. // Surf. Sci. 2010. Vol. 604. P. 476-477. DOI: 10.1016/j.susc.2009.11.030

[5] Chaplin M. Water structure and science [Электронный ресурс]. Режим доступа:

www1. 1sbu.ac.uk/water/ water_structure_science.html

[6] Verwey E.J.W., Overbeek J.Th.G. Theory of the stability of lyophobic colloids. Elsevier, 1948. 205 p.

[7] Yarom M., Marmur A. // Langmuir. 2015. Vol. 31. P. 7792 7798. DOI: 10.1021/acs.langmuir.5b00715

[8] Zargarzadeh L., Elliott J.A.W. // Langmuir. 2016. Vol. 32. P. 11309-11320. DOI: 10.1021/acs.langmuir.6b01561

[9] Koshoridze S.I., Levin Yu.K. // Nanosci. Technol. 2019. Vol. 10. N 1. P. 21-27.

DOI: 10.1615/NanoSciTechnolIntJ.2018028801

[10] Lohse D., Zhang X. // Phys. Rev. E. 2015. Vol. 91. 031003. DOI: 10.1103/PhysRevE.91.031003

[11] Kameda N., Sagashi S., Nakabayashi S. // Surf. Sci. 2008. Vol. 602. P. 1579-1584.

[12] Svetovay V.B., Devićl, Snaeijer J.H., Lahse D. // Langmuir. 2016. Vol. 32. P. 1188-11196.

DOI: 10.1021/acs.langmuir.6b01812 